\title{
Improved radiation thermometry measurement uncertainty through implementing a primary scale in an industrial laboratory
}

\author{
Jon R. Willmott ${ }^{1}$, David Lowe ${ }^{2}$, Mick Broughton ${ }^{3}$, Ben S. White ${ }^{1}$ and Graham Machin ${ }^{2}$ \\ ${ }^{1}$ University of Sheffield, Mappin Street, Sheffield S1 3JD UK \\ ${ }^{2}$ National Physical Laboratory, Hampton Road, Teddington, TQ11 OLW UK \\ ${ }^{3}$ Land Instruments International Ltd, Stubley Lane, Dronfield, S18 1DJ UK
}

\begin{abstract}
Résumé. Une échelle de température primaire nécessite la réalisation d'une unité à partir de sa définition. Pour la thermométrie de radiation, aux températures les plus élevées, dans l'Échelle internationale de température de 1990, cela consiste à extrapoler à partir du signal mesuré à la température de congélation de l'or, d'argent ou de cuivre en utilisant la loi de rayonnement de Planck. La difficulté induite est liée à la nécessité d'un équipement spécialisé caractérisé minutieusement afin de parvenir à l'extrapolation avec une précision suffisante. En tant que tel, la réalisation de l'échelle à des températures élevées est habituellement seulement pratique pour les instituts nationaux de métrologie ; les laboratoires d'étalonnage doivent compter sur une copie de l'échelle. A des températures plus basses, il est pratique pour un laboratoire d'étalonnage industriel d'avoir sa propre réalisation de l'échelle de température primaire, ce qui réduit le nombre d'étapes entre l'échelle principale et l'utilisateur final. Les modifications proposées au SI qui introduiront des points fixes de référence à haute température, internationalement reconnus, pourraient rendre pratique d'avoir une échelle de température élevée primaire dans un laboratoire d'étalonnage. Dans cette étude, une telle échelle a été établie par l'étalonnage de pyromètres directement aux points fixes de référence à haute température. La réduction de l'incertitude accessible à un utilisateur final à la suite de ce raccourcissement de la chaîne de traçabilité a été évaluée.
\end{abstract}

\section{Introduction}

Many diverse industrial processes involve temperature measurement above $1000{ }^{\circ} \mathrm{C}[1,2,3]$. Improvement to metrology in this area could lead to reduced energy usage, and tighter process control could lead to more consistent product quality. At high temperatures radiation thermometers for non-contact measurement are commonly used. Traceable temperature measurement starts with a primary realisation of the International Temperature Scale of 1990 (ITS-90) [4]. In the high temperature regime this is currently based on extrapolation from a reference of the freezing temperature of silver, gold or copper. At temperatures below the freezing temperature of silver $\left(961.78{ }^{\circ} \mathrm{C}\right)$ it is realistic for an industrial calibration laboratory to have a primary temperature scale realisation, that is, a temperature scale based on the specified methodology given in the text of the ITS-90. At higher temperatures it is impractical for all but National Measurement Institutes (NMI) to maintain a full ITS-90 temperature scale and so have the lowest achievable uncertainties. There is typically a sequence of calibration steps from NMI to industrial calibration laboratory to industrial end user. Practicalities dictate that commercial radiation thermometers are at least four and more usually six steps removed from the basic temperature scale definition. Each time the scale is transferred in this way, measurement uncertainty increases.

Proposed changes to the SI and the mise en pratique for the definition of the kelvin (MeP-K) [5] may soon make it practical for industrial calibration laboratories to have their own primary realisation of an internationally agreed thermodynamic equivalent to the ITS-90. A series of high temperature fixed points (HTFP) [6] of specified temperatures and uncertainties will allow an interpolated scale from the silver point up to at least $2500{ }^{\circ} \mathrm{C}$. A set of fixed point cells and a radiation thermometer as the interpolation device will be sufficient for maintaining the scale, and so will be practical outside the NMI. However, end users of radiation thermometers will only benefit from the changes if the industrial calibration laboratory primary standard thermometers are of an appropriate design that balances the calibration uncertainty budgets correctly. In this study, we examine the effects upon measurement uncertainty between transferring the fundamental temperature scale realised at the industrial calibration laboratory to the end user using different radiation thermometers.

HTFP of cobalt-carbon, platinum-carbon and rhenium-carbon were used in a high temperature furnace to provide a series of calibration points. These were used to calibrate firstly a bespoke designed reference-standard industrial radiation thermometer and then the same type 
of instrument modified to give a narrower spectral response and improved stability. Finally a radiation thermometer specifically designed for use in this manner was calibrated. The effect of instrument choice and the number and type of fixed points used on the calibration chain could then be evaluated.

\section{Experimental}

The measurements were carried out using the facilities [7] of the calibration laboratory at Land Instruments International Ltd. (LAND). They were the UK's first industrial ISO 17025 UKAS accredited laboratory for thermal measurements and it is an example of a laboratory that maintains its own ITS-90 scale below the silver point and currently relies upon NPL traceability above it. LAND has issued over 21,000 calibration certificates in the laboratory's 45 years of operation and they design their own bespoke standard radiation thermometers. These factors combined enable LAND to report particularly small uncertainties for an industrial laboratory, not only to its end users, but in support of LAND's wider manufacturing facility. In our study, NPL supplied the fixed points, the furnace tubes and the insulation used to optimise thermal conditions in the furnace. NPL, LAND and University of Sheffield (UoS) each supplied a radiation thermometer; denoted ATK (NPL), S1-LII (Land), S1-UoS (Modified) respectively. The two S1 thermometers were identical except for the modifications deemed necessary in order to demonstrate the benefits of the new MeP-K temperature scale realisation.

Measurements were made over three days, with each day devoted to either rhenium-carbon, platinum-carbon or cobalt-carbon, and the two S1 instruments, manufactured by LAND, were measured at the three fixed points. The ATK radiation thermometer relied on measurements of HTFPs made previously at NPL.

The three radiation thermometers were then compared directly to each other at NPL, and indirectly to the NPL primary scale via the ATK.

Finally the thermometers were used to assign a temperature with uncertainty to a furnace operating at the $\mathrm{UoS}$ in order to demonstrate the benefits of the MeP-K to end users of radiation thermometers.

\subsection{Fixed point cells}

The fixed point cells were graphite crucibles, $24 \mathrm{~mm}$ in diameter, $40 \mathrm{~mm}$ long and had a $3 \mathrm{~mm}$ diameter blackbody cavity enclosed in an ingot of metal-carbon alloy at the eutectic composition, with a volume of about $3 \mathrm{~cm}^{3}$. The melting temperature values used were those specified in [8]. Different fixed-point cells were used for the ATK and for the two S1 based devices. Allowance was made for non-reproducibility between cells in the uncertainty budget, the dominant contributions were the result of impurities and differences in thermal conditions between furnaces.

The fixed-point cells were pre-set up at NPL in furnace tubes with suitable insulation. This was to simplify the measurement process, rather than any difficulty in setting up at the LAND laboratory, and such insulation is routinely supplied with HTFP purchased from NPL. Installation was then simply a matter of removing the furnace calibration tube and replacing with the appropriate fixed-point cell assembly.

\subsection{Furnace}

The calibration laboratory at LAND uses a Thermogauge [9] $25.4 \mathrm{~mm}$ dual blackbody calibration source. It operates with an argon purge so does not need any window to protect/seal the graphite within an inert atmosphere. The radiation thermometer is aligned on the $3 \mathrm{~mm}$ fixed point cell cavity by eye and/or by scanning to identify the radiance profile when hot. The fixed point assemblies were heated and cooled at approximately 15 $20^{\circ} \mathrm{C} / \mathrm{min}$

\subsection{Radiation Thermometers}

LAND's S1 are high quality radiation thermometers, based on standard products [10], and bespoke designed as working standards for calibrating other thermometers within the production environment. Their traceability is through NPL, though, as previously described, they are two calibration steps removed from ITS-90. Two radiation thermometers from the S1 range, S1-LII and S1UoS were developed specifically for use with HTFPs in a LAND-NPL collaborative project around fifteen years ago [11]. The calibration history of these instruments has since been tracked and it was found that the design is remarkably stable, with no significant drift in calibration over the intervening years. Prior to modification of S1UoS, these two S1 instruments were identical and, therefore, provided an excellent testbed for our study.

\subsubsection{Unmodified S1-LII}

The S1-LII was unmodified since its original manufacture. It had a spectral response defined by an RG850 filter and the detection limit of the silicon photodiode detector. The size of the semiconductor bandgap of silicon is inherently temperature dependent, which means the output was substantially affected by changes in the internal temperature of the instrument. It was not temperature stabilised but relied on characterisation of the signal drift with temperature, followed by numerical ambient temperature correction. However, this design gave the instrument high optical throughput compared to the narrow-filter NMI thermometers and excellent signal to noise ratio at low temperatures.

\subsubsection{Modified S1-UoS}

The S1-UoS was modified in two ways. Firstly, the RG850 filter was replaced with an interference filter [12]. Almost all the changes in the detector photocurrent as a function of ambient temperature were as a result of the sensitivity to long wavelengths changing. The 
interference filter stabilised the photocurrent by removing these wavelengths from the measurement. A relatively narrow spectral filter was used such that the thermometer could now be considered pseudo-monochromatic. From Planck's Law, temperature is a function of radiant intensity and also wavelength; by operating at a single wavelength, the modified S1 could be used as an interpolation device between the fixed points. Unfortunately, interference filters themselves drift with ambient temperature and in order to stabilise this drift, the detector housing assembly was wrapped in resistance wire and a PID controller was used to stabilise the detector and filter metal housing at $40{ }^{\circ} \mathrm{C}$, by means of a thermistor mounted within the assembly.

\subsubsection{ATK}

The third radiation thermometer used in this study was designed at NPL and its design details are described elsewhere [13]. The instrument was designed to simplify establishing a low uncertainty scale. The optics gave small target size and it had a fairly complicated optical assembly that provided good size-of-source response. The detector and amplifier were specifically chosen for linearity and stability. The coloured glass filter was chosen to be sufficiently narrow band spectral response to give minimum deviation from the Planck response while also being stable and having low temperature coefficient. An interference filter could have been specified if required. The optical and electronic components were temperature stabilised using resistive heater pads and the mechanical structure had matched thermal expansions to keep a constant optical geometry, regardless of environmental conditions. It was intended to be calibrated with HTFPs and then used as a low uncertainty reference standard thermometer.

\subsection{Temperature scale}

General purpose radiation thermometers have a wide spectral response, required in order to achieve good signal to noise ratios at low temperature. The temperature scale that they carry is through transfer-standards with very narrow responses, which follow Planck's Law with lower uncertainty. This is presently how a primary scale at an NMI is transferred to calibration laboratories.

To set up what will be classed a primary scale for this study after calibration at the fixed points the results were fitted to an interpolation function based on Planck's law, the Sakuma-Hattori equation [14]

$$
S_{i n t}=C\left[\exp \left(\frac{c_{2}}{A T+B}\right)-1\right]^{-1}
$$

There are three adjustable parameters $(A, B, C)$ and $\mathrm{c}_{2}$ is Planck's second radiation constant, which has a value of $0.014388 \mathrm{~m} \mathrm{~K}$. With three fixed point measurements the fitting is exact. To simplify the scale fitting process, one of the parameters can be calculated. It has been shown [15] that the $B$ parameter, which is related to the width of the bandpass spectral response, has only a weak effect upon $S_{\text {int. }}$ A reasonable value for $B$ can, therefore, be calculated if the spectral response is not too wide. The manufacturer's data for filter transmission together with the sensitivity of the photodiode can be integrated over the whole spectrum to calculate a signal to temperature response. A fit to three or more selected calculated data points then gives the three adjustable parameters. One of these, $C$, relates to the overall response, so needs detailed knowledge of lens transmission, geometric factors and amplifier gain and cannot be taken from the integration results. Within ITS-90 above the silver point this detailed knowledge is largely by-passed by making all measurements relative to one of the defined fixed-points. The parameter $A$ gives the central wavelength of the spectral response and the calculated temperature for a given signal is very sensitive to small changes in its value, so again the calculated value cannot be used without extensive further characterisation. Parameter $B$, however is relatively insensitive: for a narrow interference filter a simple "by-eye" evaluation of the filter spectral response curve can be sufficient to estimate $B$ with insignificant error. [15] The calculated value for this parameter can therefore be inserted directly into (1), meaning that two fixed points would be sufficient for a complete calibration according to MeP-K.

\subsection{Uncertainty budgets}

The uncertainties propagate in a fairly complex manner, and at each temperature there is a contribution from both uncertainty in the reference points' temperatures and from the signals from the instrument. Fortunately, the propagation equations for any number of fixed points are known [16]. The fixed-point temperature uncertainty is intrinsic to the fixed point cell itself. It contains components for the knowledge of the pure ingot melting temperature [8], effect of impurities, temperature drop across the fixed point cell cavity wall, the identification of the point at which the fixed point is judged to be at the melting temperature and an allowance for the intrinsic repeatability of the transition [17]. Allowance was also made for possible variation due to different thermal conditions in different furnaces and complications arising from the very fine alloy microstructure with consequent large interfacial energy effects.

There is then uncertainty in the actual instrument signal as it measures the fixed points: the uncertainty intrinsic to the thermometer. This includes ambient effects, the emissivity of the source (this depends on wavelength so is included here as an instrument component), the optical quality (judged from size-ofsource effect), the current to voltage conversion and the repeatability of the measurements.

The fractional signal uncertainty can then be expressed in temperature terms via

$$
\Delta T=\frac{\lambda T^{2}}{c_{2}} \frac{\Delta I}{I}
$$

The combination of the propagated uncertainties from the calibration points is then combined with uncertainties 
associated with the actual use of the instrument: the effect of ambient conditions, size-of-source-effect, current to voltage conversion, stability and non-linearity. This uncertainty is intrinsic to the instrument in a given measurement situation, here it is the calibration comparison conditions at NPL.

There should be an additional included uncertainty: the departure from the ideal Planck behaviour. In this case, though, we are interested in how well the thermometers perform as standards and the extent to which they agree, and so this evaluation has not been carried out as expanding the uncertainties might mask an effect that would be useful to observe.

\subsection{Scale comparison}

When the fixed point measurements were evaluated equation (1) was fitted to the results and the uncertainty budgets were calculated. The values used for temperature for the fixed-point cells were the best available thermodynamic melting temperatures. Each thermometer therefore carried what will be, once the expected changes to the $\mathrm{MeP}-\mathrm{K}$ are incorporated, a primary scale. The three thermometers were compared at NPL from $1000{ }^{\circ} \mathrm{C}$ to $2600{ }^{\circ} \mathrm{C}$ using a Thermogauge dual blackbody similar to that at used at LAND. In addition the ATK instrument was compared to the main NPL primary ITS-90 realisation [18].

\subsection{Validation of LAND primary scale}

With their own primary scale LAND would be in a position to supply an instrument directly calibrated to it, which would be expected to offer very low uncertainties. In order to demonstrate the benefits to end users, measurements were made at the UoS with all three instruments measuring the temperature of a three-zone furnace.

\section{Results}

The S1 both modified and unmodified were used to successfully demonstrate that a practical temperature scale above the silver point can be realised at an industrial laboratory with low uncertainty. With all the measurement uncertainties evaluated and combined, we find that the ATK, which was specifically designed for low uncertainty under a study of this methodology, was the more accurate.

The high volume production instrument most similar to the $\mathrm{S} 1$ [10] has a measurement uncertainty guaranteed to be lower than $8{ }^{\circ} \mathrm{C}(0.7 \% \mathrm{~K})$ whilst measuring a target of $2000^{\circ} \mathrm{C}$. As shown in figure 1 , the HTFP calibration methodology improves the measurement uncertainty by a factor of six compared to commercial thermometers. Whilst the improvement was huge, it should be noted that it is too time consuming to calibrate high volume production instruments using HTFPs. Further, it is not known before manufacture how accurate a thermometer will eventually be and the data sheet specifications are worst case, whereas, the data in figure 1 are measured for individual instruments. A small improvement to measurement uncertainty was achieved by the modifications to the $\mathrm{S} 1$. These were in part due to the improved temperature stability and also due to the shorter effective wavelength at which the modified instrument measured temperature.

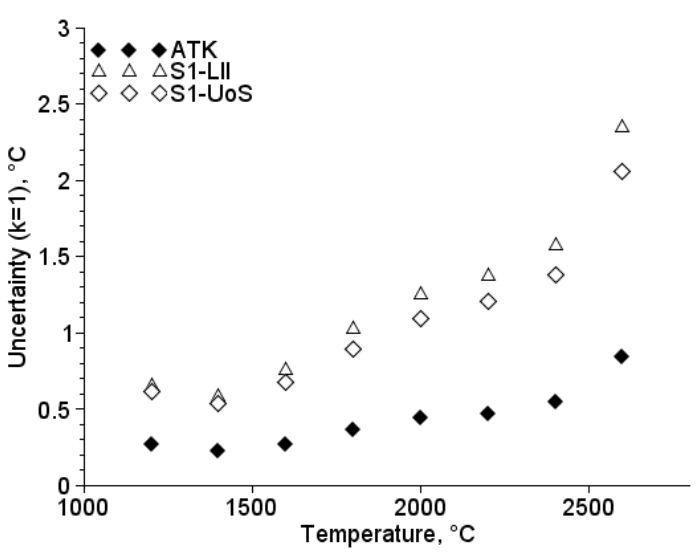

Figure 1. Calibration uncertainty for the three instruments.

In the second part of the experiment, the thermometers were each taken to NPL in order to compare them with NPL's primary ITS-90 scale and the results are shown in figure 2. There was little difference between the measurements of the ATK and S1-LII, except that the latter exhibits a slight positive offset.

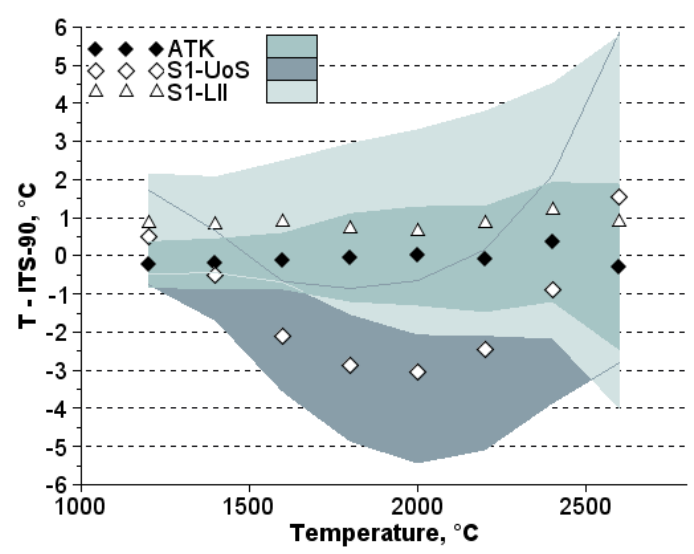

Figure 2. Comparison of the three primary scales to the ITS-90 primary scale at NPL. Shaded areas indicate the comparison uncertainty for each instrument at $k=2$.

The S1-UoS made some accurate measurements at low and high temperature, however it measured low between these points. In spite of this, it was clear that compared to a typical commercial thermometer, all three instruments carried with them a directly realised temperature scale that could be maintained outside an NMI and measured with good accuracy.

In order to demonstrate the advantages of the MeP-K for end users, we assessed the temperature of a pseudo- 
blackbody calibration furnace at UoS. The furnace controller measured the temperatures of internal thermocouples and the measurements were not necessarily representative of the radiance temperature that the radiation thermometers measured.

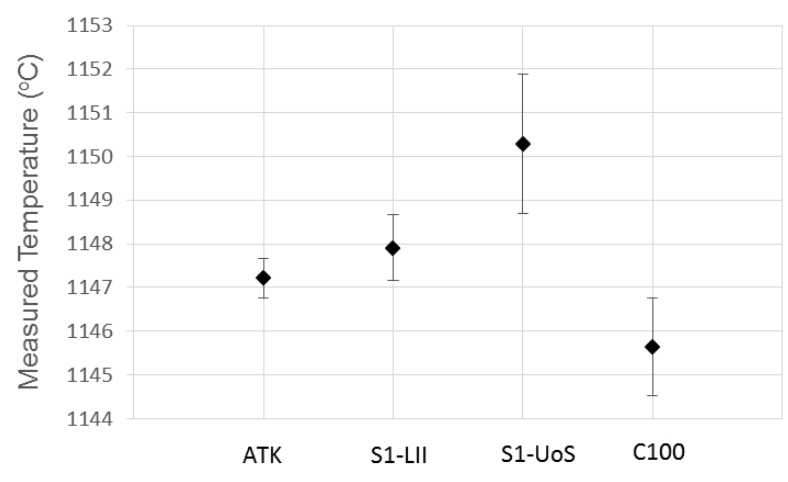

Figure 3. Measurements of an end user furnace, together with a measurement by a commercial UKAS certified radiation thermometer (C100). The furnace set point was $1150{ }^{\circ} \mathrm{C}$.

On this occasion, the thermocouple measurements were reasonable, with a value of $1150^{\circ} \mathrm{C}$ for the data presented in figure 3. A fourth measurement is also plotted and this was measured by LAND's commercial solution for laboratory calibration, the carefully calibrated and certified C100 [19]. The S1-UoS measurements are high compared to the other data, though these results demonstrate measurements from a primary scale with reasonable accuracy. The excellent performance of the S1-LII demonstrates the potential for improved uncertainties compared to the commercial solution.

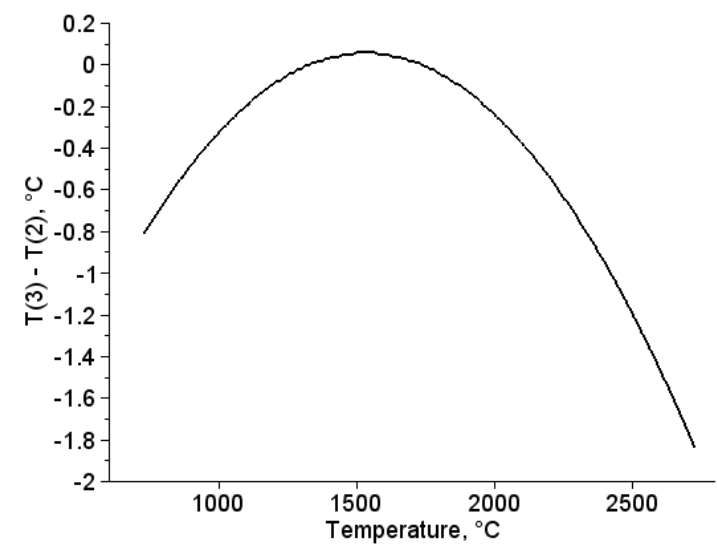

Figure 4. The difference $\mathrm{T}(3)-\mathrm{T}(2)$ in temperature scales between fitting to three fixed-points and fitting to two fixedpoints with the addtional parameter determined from readily available spectral response data of the interference filter and silicon photodiode.

Figure 4 shows the difference between the scales generated by the S1-UoS from fitting to all three fixed points compared to fitting just to cobalt-carbon and platinum-carbon. The parameter was calculated from the filter and detector.

\section{Discussion}

Technical problems with the S1-UoS instrument build lead to increased SSE and slightly reduced the transmission between fixed point measurements compared to the unmodified instrument. In spite of this, our results accurately demonstrate the benefits of the $\mathrm{MeP}-\mathrm{K}$, through improved measurement uncertainty that could be achieved by a second iteration of the build. The S1-LII performance is clearly good, though the raw thermometer output voltage is substantially less stable than the S1-UoS, with very big drifts as a hot source heats the detector and changes the spectral response. The unmodified instrument performs well here because its spectral drift with ambient temperature has been very carefully characterised at a previous date. This approach is appropriate for a large production run of many thousands: a generic behaviour is established for a model and then individual thermometers are calibrated by comparison to a standard at three temperatures and a cubic correction function is applied. The S1-LII performs better than a similarly designed commercial instrument because great lengths went into characterising the signal output and by replacing the electronic components with very expensive, low drift amplifiers and other precision components. However, we have demonstrated that this approach of characterisation is unnecessary for small production runs when the aim is excellent accuracy for calibration, rather than for general purpose industrial thermometry where low component cost and good low temperature signal to noise ratio is more of a concern. The advantage of stabilising the thermometer is that a three point calibration based on HTFP is all that is required, making it possible to make simple modifications to commercial instruments that allows a primary temperature scale to be maintained within an industrial laboratory.

The difference between the three-point and two-point calibrations is less than $1{ }^{\circ} \mathrm{C}$ from $1000{ }^{\circ} \mathrm{C}$ to $2400{ }^{\circ} \mathrm{C}$. This is a compromise that would in many cases be acceptable. It should be possible to simplify the process further by use of one of the ITS-90 fixed points and one HTFP.

\section{Conclusion}

For a calibration laboratory that has a high temperature tube furnace, we have demonstrated that the MeP-K makes the establishment of a primary scale straightforward. Some differences in working practices will require addressing, particularly the need to align the instruments on a small area of an otherwise rather uniform target. Both the thermometer types give improved uncertainties to the end user. The S1 is suitable for manufacture in high volume and could be supplied as a device calibrated against HTFPs, with low uncertainty, in a form-factor that makes it rugged and suitable for use outside the laboratory. The ATK offers lower uncertainty 
and might be more suitable to a situation where a calibration laboratory or a smaller NMI needs everyday access to an ultra-low uncertainty scale but without daily recourse to measuring HTFPs. We also showed that a 'two point fit,' where the radiation thermometer interpolates between only two HTFPs, appears to be perfectly adequate. This further simplifies calibration and allows easier optimisation of a standard radiation thermometer, in order to become compatible with HTFPs and the MeP-K.

\section{References}

[1] J. Dixon, J. Phys. E: Sci. Instrum, 21(5), 425-436 (1988)

[2] J. C. Richmond and D. P. DeWitt, American Society for Testing and Materials, 895 (1985)

[3] D. P. DeWitt and G. D. Nutter, Theory and Practice of Radiation Thermometry (John Wiley \& Sons, 1989)

[4] T. J. Quinn, Temperature (Academic Press, Second Edition, 1990).

[5] D. Ripple, R. Davis, B. Fellmuth, J. Fischer, G. Machin , T. Quinn, P. Steur, O. Tamura and D. R. White, Int. J. Thermophys, 31, 1795-808 (2010)

[6] G. Machin, P. Bloembergen, J. Hartmann, M. Sadli and Y. Yamada, Int. J. Thermophys, 28, 1976-1982 (2007)

[7] https://www.landinst.com/pages/calibration

[8] E.R. Woolliams, K. Anhalt, M. Ballico, P. Bloembergen, F. Bourson, S. Briaudeau, J. Campos, M.G. Cox, D. del Campo, M.R. Dury, V. Gavrilov, I. Grigoryeva, M.L. Hernanz, F. Jahan, B. Khlevnoy, V. Khromchenko, D.H. Lowe, X. Lu, G. Machin, J.M. Mantilla, M.J. Martin, H.C. McEvoy, B. Rougié, M. Sadli, S.G. Salim, N. Sasajima, D.R. Taubert, A.D.W. Todd, R. Van den Bossche, E. van der Ham, T. Wang, D. Wei, A. Whittam, B. Wilthan, D.J. Woods, J.T. Woodward, Y. Yamada, Y. Yamaguchi, H.W. Yoon, Z. Yuan Phil. Trans. R. Soc. A. submitted (2015)

[9] http://www.thermogauge.com/ht-5500.htm

[10] https://www.landinst.com/products/system-4-highprecision-thermometers

[11] G. Machin, G. Beynon, F. Edler, S. Fourrez, J. Hartmann, D. Lowe, R. Morice, M. Sadli and M. Villamanan, AIP, 7, 285-290 (2003)

[12] http://www.semrock.com/FilterDetails.aspx?id=FF02 $-809 / 81-25$

[13] D. Lowe, Measurement Science and Technology, 24 (2013)

[14]F. Sakuma and S. Hattori, Temperature: Its Measurement and Control in Science and Industry, $\mathbf{5}$, 421-7 (New York, AIP, 1982)

[15] P. Saunders and D. R. White Metrologia 40, 195-203 (2003)

[16]P. Saunders, Metrologia 34, 201 (2000)

[17] "Uncertainty Budgets for realization of scales by radiation thermometry" www.bipm.org/cc/CCT/Allowed/22/CCT03-03.pdf
[18] D Lowe G Machin Traceable radiation thermometer calibration to ITS-90 above the silver point at the National Physical Laboratory this conference

[19] https://www.landinst.com/products/cyclops-100Lportable-non-contact-thermometer 